\title{
Invasive breast cancer in Argentine women: association between risk and prognostic factors with antigens of a peptidic and carbohydrate nature
}

\author{
This article was published in the following Dove Press journal: \\ Breast Cancer:Targets and Therapy \\ 19 December 2011 \\ Number of times this article has been viewed
}

\author{
Sandra O Demichelis \\ Marina T Isla-Larrain \\ Luciano Cermignani \\ Cecilio G Alberdi \\ Amada Segal-Eiras \\ María Virginia Croce \\ Centre of Basic and Applied \\ Immunological Research, Faculty of \\ Medical Sciences, National University \\ of La Plata, La Plata, Argentina
}

Correspondence: Maria Virginia Croce Centre of Basic and Applied Immunological Research, Faculty of Medical Sciences, National University of La Plata, Calle 60 and I20,

1900, La Plata, Argentina

Tel +54 22I 42367II ext 342

Fax +54221 4258989

Email crocevir@hotmail.com
Objective: In breast cancer, several tumor markers have been identified. The marker most extensively associated with breast cancer is MUC1. The objective of the study was to analyze prognostic and risk factors in relation to tumor markers in order to clarify breast cancer biology. A total of 349 primary tumor samples and lymph nodes from breast cancer patients were studied. Risk and prognostic factors were considered. An immunohistochemical approach was applied and an extensive statistical analysis was performed, including frequency analysis and analysis of variance. Correlation among variables was performed with principal component analysis.

Results: All the antigens showed an increased expression according to tumor size increment; moreover, sialyl Lewis x expression showed a significant increase in relation to disease stage, whereas Tn and TF presented a positive tendency. Vascular invasion was related to sialyl Lewis $\mathrm{x}$ expression and number of metastatic lymph nodes. Taking into account risk factors, when a patient had at least one child, Lewis antigens diminished their expression. In relation to breastfeeding, sialyl Lewis x expression diminished, although its apical expression increased. Conclusion: Associations between MUC1 and carbohydrate antigens and risk and prognostic factors show the complexity of the cellular biological behavior that these antigens modulate in breast cancer.

Keywords: breast cancer, Argentine women, risk factors, prognostic factors, antigenic expression

\section{Introduction}

In Argentina, it has been estimated that 17,000 new cases of breast cancer are diagnosed each year. This tumor localization is the commonest cause of cancer death in women, reaching 5400 deaths per year, ${ }^{1}$ which is the second rate of mortality in Latin America. ${ }^{1}$ Although there is a strong public health system that covers all the expenditure in relation to cancer treatments, disadvantaged groups have high mortality rates, probably because of a lack of preventive actions.

Because MUC1 has been shown to be overexpressed and aberrantly glycosylated in breast and other carcinomas, in this report we studied this mucin and carbohydrate associate antigens.

MUC1 is a high molecular transmembrane glycoprotein expressed as a stable heterodimer after translation of a single polypeptide and cleavage into two subunits in the endoplasmic reticulum. ${ }^{2}$ The MUC1 N-terminal subunit contains variable numbers of 20 amino acid tandem repeats that are extensively modified by $O$-linked glycans. ${ }^{3}$ 
The MUC1 C-terminal subunit consists of a 58 amino acid extracellular domain, a 28 amino acid transmembrane domain, and a 72 amino acid cytoplasmic chain. ${ }^{4}$

MUC1 expression in normal epithelia can be quite dynamic, varying in response to steroid hormone or cytokine influences. ${ }^{5}$ Following malignant transformation, MUC1 often becomes highly overexpressed, loses its apical polarization, and displays aberrant glycosylation as well as altered mRNA splicing variants. ${ }^{6}$ In addition, post-translational regulation of cell surface expression occurs via the activity of cell surface proteases that release soluble forms of MUC1 large ectodomain. In cancer, mucin $O$-glycosylation is characterized by aberrant expression of immature carbohydrate structures such as Tn, T, and sialyl Tn antigens ${ }^{7}$ and Lewis blood group antigens (Lewis x, Lewis y, Lewis b, Lewis a, and their sialylated derivatives) ${ }^{8}$

Some reproductive factors, including age at menarche, age at first full-term pregnancy, number of live births, and breastfeeding, have been related to risk of breast cancer, ${ }^{9}$ because their impact on lifetime number of ovulatory cycles modifies exposure to endogenous ovarian hormones. ${ }^{8}$ Additionally, both pregnancy and breastfeeding induce the differentiation of breast epithelial cells, making them less susceptible to carcinogenic insults. ${ }^{10}$

MUC1 expression in breast tissue increases during pregnancy and lactation; ${ }^{11}$ also, MUC1 serum levels augment according to pregnancy development. ${ }^{12,13}$ In relation to this, it has been hypothesized that, during pregnancy, MUC1 may stimulate the immune system, which may play a protective role against breast cancer. ${ }^{14}$ MUC1 has been detected to be bound to immunoglobulins (Igs), forming circulating immune complexes (MUC1CIC) in pregnant women. ${ }^{15}$

Many authors have described associations between MUC1 expression and prognostic factors in breast cancer. In general, a significant positive correlation has been found between estrogen receptor (ER) status and MUC1 expression. ${ }^{16}$ At the same time, between increased MUC1staining and histological grade, a significant, inverse relationship was detected, ${ }^{16-18}$ as well as with a nuclear grade. ${ }^{19}$

This study selected MUC1 and glycan epitopes to ascertain their associations with different risk and prognostic factors in breast carcinoma in Argentine women.

\section{Materials and methods}

\section{Patients and tumors}

From January 2007 to December 2010, 349 patients underwent surgery for invasive breast carcinoma at institutions related to the Faculty of Medical Sciences of the National
University of La Plata, La Plata, Argentina. All these patients were included in the present study. Primary tumor samples were obtained and typed as $81 \%$ not otherwise specified ductal type (NOS ductal), 16\% lobular type (L), and $3 \%$ medular type. Tumors corresponding to NOS ductal presented different grades of differentiation: $17.2 \%$ were well differentiated, 55.2\% moderately differentiated, and $27.6 \%$ poorly differentiated. Staging according to the Union for International Cancer Control's TNM classification system was established: 105 of $349(30 \%)$ corresponded to Stage I, 142 of 349 (41\%) to Stage II, 87 of $349(25 \%)$ to Stage III, and 15 of $349(4.3 \%)$ to Stage IV. In some cases, metastatic lymphatic nodes were also obtained.

Patients' clinical data and cancer characteristics included some risk and prognostic factors. Risk factors were age at diagnosis (56 \pm 14 , range 29-85 years); age at menarche (12.9 \pm 1.5 , range 10-16 years); menopausal status: premenopausal included $43 \%$ of patients while postmenopausal, 57\%; breastfeeding: positive $51.7 \%$ and negative $48.3 \%$; and children: positive $74.3 \%$ and negative $25.7 \%$. Prognostic factors included disease stage; axillary nodal status: negative (1) $38.7 \%$ of patients, positive: $\leq 3$ (2) $84.6 \%$ while $>3$ (3) $15.4 \%$, not assessed: $1.9 \%$ of patients; tumor size: T1 $38.1 \%$ of patients, T2 $54.3 \%$, and T3 $7.6 \%$; histological differentiation: I $31.5 \%$, II $45.7 \%$, and III $22.8 \%$; nuclear grade: Grade $132.7 \%$, Grade $240.4 \%$, and Grade $326.9 \%$; mitotic figure counts; Nottingham Prognostic Grade: ${ }^{20}$ good (I) $44.6 \%$, moderate (II) $42.9 \%$, and poor (III) $12.5 \%$; vascular invasion: positive $29.8 \%$ and negative $70.2 \%$; ER status: positive $66.7 \%$, negative $18.1 \%$, unknown $15.2 \%$; and progesterone receptor (PR) status: positive $63.8 \%$, negative $18.1 \%$, unknown $18.1 \%$.

\section{Controls}

A total of 148 tissue samples belonging to patients with benign breast disease (BBD) and normals were included. BBDs were classified as follows: (1) simple fibroadenoma (FA), (2) nonproliferative lesions (NPF) comprised fibrocystic changes/apocrine metaplasia and fibrosis, (3) proliferative lesions without atypia (usual epithelial hyperplasia [UEH]), and (4) proliferative lesions of ductal type with atypial ductal hyperplasia (ADH).

A total of 116 samples corresponded to BBD being $50 \mathrm{FA}$, $28 \mathrm{NPF}, 30 \mathrm{UEH}$, and eight ADH. Also, 32 normal specimens obtained during breast cosmetic surgery were included. The mean age of patients with BBD and normal females was $42.2 \pm 13.6$ years. 
Procedures followed the World Medical Association Declaration of Helsinki (Finland, 1964) and further modifications. Informed consent was obtained from all patients included in this study. This research was approved by the local Human Investigation Committee, Faculty of Medical Sciences, National University of La Plata.

Tumors and tissues were collected fresh and delivered to the laboratory with minimal delay. For histopathological diagnosis and immunohistochemical analysis, a sample fraction was fixed in methacarn (methanol: chloroform: acetic acid 60:30:10) for 2 hours and transferred into $70 \%$ ethanol until processing in paraffin.

\section{Monoclonal antibodies (Table I)}

Three anti-MUC1 variable number of tandem repeats (VNTR) monoclonal antibodies (MAbs) were assayed: HMFG1, ${ }^{21}$ C595, ${ }^{22}$ and SM3. ${ }^{11}$ Also, five anticarbohydrate epitopes: anti-sialyl Lewis x (KM93), ${ }^{23}$ anti-Lewis x (KM380), ${ }^{23}$ antiLewis y (C14), ${ }^{24}$ anti-Tn Mabs, and anti-TF MAbs (DAKO, Copenhagen, Denmark) were used in these studies.

\section{Methods}

\section{Immunohistochemical analysis}

The technique was performed following previous reports. ${ }^{25}$ Dewaxed sections were placed in methanol with hydrogen peroxide (3\%) for 15 minutes to block endogenous peroxidase activity. After three washes in phosphate buffered saline (PBS), sections were blocked for nonspecific binding with normal horse serum diluted 1:10 in $1 \%$ bovine serum albumin/PBS for 15 minutes and rinsed. Before samples were immunostained with MAbs, tissues were treated with $10 \mathrm{mM}$ sodium citrate buffer at $100^{\circ} \mathrm{C}$ for 5 minutes for antigenic retrieval. Then, sections were incubated overnight at $4^{\circ} \mathrm{C}$ with MAbs, which were diluted as follows: C595, KM93, KM380, and C14, 1/1000; anti-Tn; and TF 1/100. HMFG1 and SM3 were not diluted (tissue culture supernatants). After three washes with PBS, peroxidase-conjugated antimouse Igs (Sigma-Aldrich Co, St Louis, MO) were added and incubated for 60 minutes. After being washed, slides were counterstained with hematoxylin and coverslipped with mounting media. In every assay, we included a positive control sample, and the same tissue section was incubated with PBS instead of the primary $\mathrm{Ab}$ as a negative control.

Specimens were examined with a light microscope and the antibody staining patterns were scored in a semiquantitative manner. Staining intensity was graded as negative, low, moderate, and strong. The number of low power $(\times 10)$ optical fields in a specimen with a positive reaction was expressed as a percentage of the total number of optical fields containing tissue. The pattern of reaction was classified as linear (membrane), cytoplasmic, and mixed pattern (cytoplasmic with plasma membrane staining), and apical and nonapical staining was recorded. ${ }^{25}$ The positive reaction of the nucleus was considered, as well as the staining of gland lumen content, which was identified as cellular debris or secretion.

\section{Statistical analysis}

Patients were grouped, taking into account risk and prognostic factors, and positive responses were evaluated by means of frequency analysis $\left(\chi^{2}\right.$ test, $\left.P<0.05\right)$.

A reactivity index $(\mathrm{RI})$ was calculated as intensity $(\mathrm{I}) \times$ $100+$ percentage of positive area (A) ${ }^{26}$

Normality of variables was tested by Shapiro Wilk's, and data were normalized when necessary.

Correlation among variables was performed with principal component analysis (PCA). Immunohistochemical pattern, percentage of positive area, and RI were analyzed employing analysis of variance $(P<0.05)$ after data standardization.

Also, logistic regression analysis was performed. In this sense, a multiple logistic regression analysis was conducted, subgrouping breast cancer patients according to ER, PR,

Table I Antibodies assayed

\begin{tabular}{|c|c|c|c|c|}
\hline Antigen & Epitope structure & MAb & Isotype and source & Reference \\
\hline MUCIVNTR & Pro-Asp-Thr-Arg & HMFGI & $|g G|$ - mouse $M A b$ & Taylor-Papadimitriou J et $\mathrm{al}^{21}$ \\
\hline MUCIVNTR & Arg-Pro-Ala-Pro & C595 & $\lg G 3$ - mouse $M A b$ & Price et $\mathrm{al}^{22}$ \\
\hline MUCI VNTR & Pro-Asp-Thr-Arg-Pro & SM3 & $\operatorname{lgGI}$ & Burchell et al'" \\
\hline Sialyl Lewis $\mathrm{x}$ & NeuAc2-3Gal $\beta$ I-4GlcNAc $\beta$ I-3Gal-R & KM93 & IgM - mouse MAb & Hanai et $a^{23}$ \\
\hline Lewis $x$ & Gal $\beta$ I-4GIcNAc $\beta \mid-3 G a l-R$ & KM380 & IgM - mouse $M A b$ & Hanai et $\mathrm{al}^{23}$ \\
\hline Lewis y & $\begin{array}{l}\text { Gal( } \alpha \text { l-2Fuc }) \beta I-4 G l c N A c(\alpha \mid-3 F u c) \\
\beta I-3 G a l-R\end{array}$ & $\mathrm{Cl} 4$ & $\lg M-$ mouse $M A b$ & Brown et $\mathrm{al}^{24}$ \\
\hline Tn & GalNAco-R & Anti-Tn & $\lg M-$ mouse $M A b$ & Dako $^{\mathrm{a}}$ \\
\hline Thomsen-Friedenreich & Gal $\beta I-3 G a l N A c \alpha \mid-R$ & Anti-TF & IgM - mouse $M A b$ & Dako $^{a}$ \\
\hline
\end{tabular}

Notes: aDakopatts, Dako Corporation, Copenhagen, Denmark. Abbreviations: Ig, immunoglobulin; MAb, monoclonal antibody. 
Table 2 Percentage of positive immunohistochemical results obtained in normal, benign, and cancer breast samples

\begin{tabular}{|c|c|c|c|c|c|c|c|c|}
\hline \multirow[t]{2}{*}{ Specimens } & \multicolumn{3}{|c|}{ Anti-VNTR MUCI } & \multicolumn{5}{|c|}{ Anti-carbohydrate antigens MAbs } \\
\hline & C595 & HMFGI & SM3 & Lewis $\mathrm{x}$ & sialyl Lewis $\mathrm{x}$ & Lewis y & Tn & TF \\
\hline Normal & 40 & 100 & 5 & 46 & 10 & 7 & 2 & 0 \\
\hline FA & 57 & 92 & 0 & 50 & 12 & 3 & 21 & 2 \\
\hline NPF & 58 & 100 & 2 & 58 & 15 & 25 & 10 & 3 \\
\hline UEH & 56 & 100 & 16 & 92 & 24 & 28 & 36 & 14 \\
\hline $\mathrm{ADH}$ & 57 & ND & 14 & 71 & 14 & 43 & 14 & 29 \\
\hline I & 63 & 92 & 40 & 54 & 24 & 22 & 25 & 3 \\
\hline II & 59 & 92 & 38 & 57 & 25 & 31 & 31 & 22 \\
\hline III & 58 & 93 & 41 & 44 & 35 & 20 & 35 & 28 \\
\hline IV & 86 & 98 & 47 & 60 & 33 & 40 & 20 & 49 \\
\hline
\end{tabular}

Abbreviations: ADH, atypial ductal hyperplasia; FA, fibroadenoma; MAbs, monoclonal antibodies; NFP, nonproliferative lesions, UEH, usual epithelial hyperplasia; VNTR, variable number of tandem repeats.

and Her2-neu positive-negative responses using patients' prognostic and risk factors as independent variables. Missing data cases were deleted (minimum $n=332$ ).

\section{Results}

Immunohistochemical reactivity of the different antigens assayed obtained in malignant samples according to disease stage are summarized in Table 2; results obtained in control samples (benign and normal) are also included. Results are expressed as percentage of positive samples in respect of the total.

Table 3 shows antigenic expression results of normal breast, $\mathrm{BBD}$, and breast cancer samples expressed as RI.

\section{Breast cancer}

\section{$\mathrm{MUCl}$ expression in primary tumor samples}

MUC1 expression was detected employing three anti-MUC1 VNTR MAbs. Most samples were stained with at least one anti-MUC1 MAb; the most reactive was HMFG1 MAb, because $92.7 \%$ of samples were positive, whereas C595 MAb reacted with $61.4 \%$ of samples and SM3 MAb with 39.5\% of samples.

As has been previously observed, ${ }^{17,18,27}$ the cellular localization as well as the intensity of the staining varied among specimens. Most samples showed a nonapical, cytoplasmic pattern with a strong staining (Figure 1A, B, and C), and several samples showed an apical pattern with a linear, cytoplasmic, or mixed reaction.

\section{Carbohydrate antigenic expression in primary tumor samples}

Seventy-three percent of samples expressed at least one glycan antigen. Lewis $\mathrm{x}$ was the most frequently found, and $50.7 \%$ of tumors showed reactivity with either a linear or a mixed staining, mainly restricted to the apical part of the ductal cells. In most cases, a strong reaction was detected.

In respect of sialyl Lewis $x$, it was found that $27.5 \%$ of samples were reactive. Staining was mainly linear and apical, although some samples depicted a cytoplasmic nonapical reaction (Figure 1D). Twenty-six percent of specimens were positive for Lewis y, showing different patterns of staining: linear and apical in some samples and cytoplasmic and nonapical in some others, whereas several specimens showed a mixed and apical or even nonapical staining. Tn hapten was detected in $29.6 \%$ of specimens. Reaction was mainly found in a few cells. In some tumors, expression was cytoplasmic, whereas others stained the plasmatic membranes, usually

Table 3 Antigenic expression of normal breast, BBD, and breast cancer samples expressed as reactivity indexes

\begin{tabular}{llll}
\hline Antigen & Normal samples & BBD samples & Breast cancer \\
\hline MUCI (HMFGIMAb) & $110 \pm 164$ & $162 \pm 162$ & $376 \pm 112$ \\
MUCI (C595 MAb) & $125 \pm 155$ & $185 \pm 174$ & $299 \pm 101$ \\
MUCI (SM3 MAb) & $20 \pm 70$ & $17+168$ & $261 \pm 146$ \\
Sialyl Lewis X & $27 \pm 65$ & $50 \pm 110$ & $219 \pm 109$ \\
Lewis X & $181 \pm 133$ & $234 \pm 155$ & $307 \pm 77$ \\
Lewis y & $24 \pm 68$ & $84 \pm 127$ & $269 \pm 65$ \\
Tn & $12 \pm 62$ & $46 \pm 92$ & $277 \pm 118$ \\
TF & 0 & $19 \pm 66$ & $223 \pm 129$ \\
\hline
\end{tabular}

Abbreviations: BBD, benign breast disease; MAb, monoclonal antibody. 


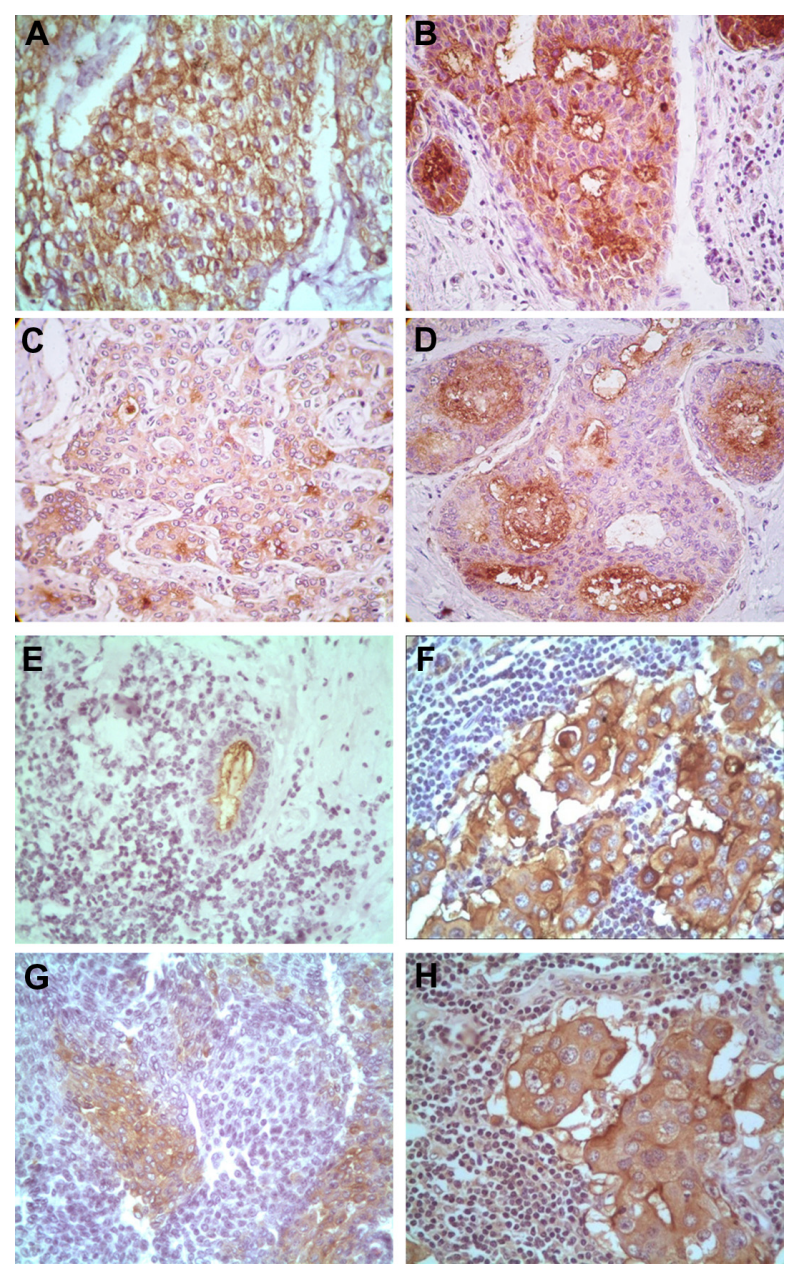

Figure I Photomicrographs of immunoperoxidase staining of primary ductal breast carcinoma samples (A-E) are shown. Staining with anti-MUCI HMFGI monoclonal antibodies (MAbs) (A), a mixed nonapical pattern of reaction with a strong intensity is observed; (B) a mixed reaction is shown (note the strong apical pattern); (C) staining with anti-MUCl C595 MAb, a cytoplasmic and moderate reaction is depicted; (D) reaction obtained with anti-sialyl Lewis $x$ MAb, a mixed reaction is shown; lumen content appears strongly stained; $(E)$ the TF antigenic reaction shows a linear and apical pattern. Immunohistochemical reactions of metastatic lymphatic nodes $(\mathbf{F}-\mathbf{H})$ are depicted: $(\mathbf{F})$ staining with anti-MUCI HMFGI MAb, a strong cytoplasmic reaction is shown; (G) the sialyl Lewis $\mathbf{x}$ cytoplasmic reaction is clearly visible in many metastatic cells and $(\mathbf{H})$ staining obtained with anti-TF MAb, a cytoplasmic strong reaction is shown.

with a mild reaction. Finally, TF was found in $25 \%$ of samples with either linear or apical (Figure 1E) or mixed nonapical patterns.

\section{Antigenic expression in metastatic lymphatic nodes}

In a group of 20 patients, a metastatic node sample was analyzed. Fifty percent of lymphatic nodes were positive for MUC1. In these samples, metastatic malignant cells showed a cytoplasmic staining with a strong intensity (Figure 1F). The most reactive anti-MUC1 MAb was HMFG1. Sialyl Lewis $\mathrm{x}$ showed reactivity in eight of 20 samples (Figure 1G) and
Lewis $\mathrm{x}$ in six of 20 samples with the same pattern. Lewis $\mathrm{y}$, $\mathrm{Tn}$, and TF (Figure 1H) were detected in four of 20 samples with a cytoplamic reaction and low intensity.

\section{Correlation among antigens}

Sialyl Lewis $\mathrm{x}$ showed a positive correlation with Tn antigen $(r=0.8147 ; P<0.034)$, whereas Lewis $\mathrm{x}$ and Lewis y showed a strong and positive correlation $(r=0.92 ; P<0.016)$.

A positive tendency between MUC1 and Lewis $\mathrm{x}$ apical expression was detected. Taking into account the RI, a positive correlation between MUC1 (detected with SM3 MAb) versus Lewis $\mathrm{x}$ was found $(r=0.8830$; $P<0.022)$.

\section{Relationship between MUCI expression and prognostic factors}

MUC1 detection with the three different anti-MUC1 MAbs (HMFG1, C595, and SM3) showed some differences when they were correlated with prognostic factors.

In order to analyze in more depth the antigenic expression, RI was calculated as intensity $(\mathrm{I}) \times 100+$ percentage of positive area (A) ${ }^{26}$ According to anti-MUC1 MAb in relation to disease stage, for HMFG1, MAb RI and Stage I results were $355 \pm 155$, Stage II $386 \pm 113$, Stage III $389 \pm 98$, and Stage IV $480 \pm 71$. A raise tendency was detected but with no statistical significance (Tukey HSD; $P=0.067$ ) (Figure 3). For C595, MAb and Stage I results were $299 \pm 110$, Stage II $287 \pm 109$, Stage III $311 \pm 84$, and Stage IV $289 \pm 122$. Finally, for SM3, MAb and Stage I results were $281 \pm 156$, Stage II $267 \pm 137$, Stage III $236 \pm 147$, and Stage IV $272 \pm 112$.

Percentage of positive responses detected with all MAbs increased according to tumor size increment (Figure 2), with statistically significant C595 and SM3 MAbs reactivity $(P<0.040)$.

It was not possible to find a relationship between the pattern of expression of MUC1 and disease stages.

Furthermore, MUC1 apical expression frequency detected with HMFG1 MAb showed a significant decrease according to histological grade increment $(P<0.031)$ (Figure 3 ). The percentage of samples with apical expression for Grade 1 tumors was $37.5 \%$, for Grade 2 tumors was $25.5 \%$, and for Grade 3 tumors was $16.7 \%$; a similar observation was found with nuclear Grade I 41\%, Grade II $19 \%$, and Grade III $16 \%$.

Finally, considering the percentage of MUC1 reactive area, tumors that presented a high percentage showed a low nuclear grade. 


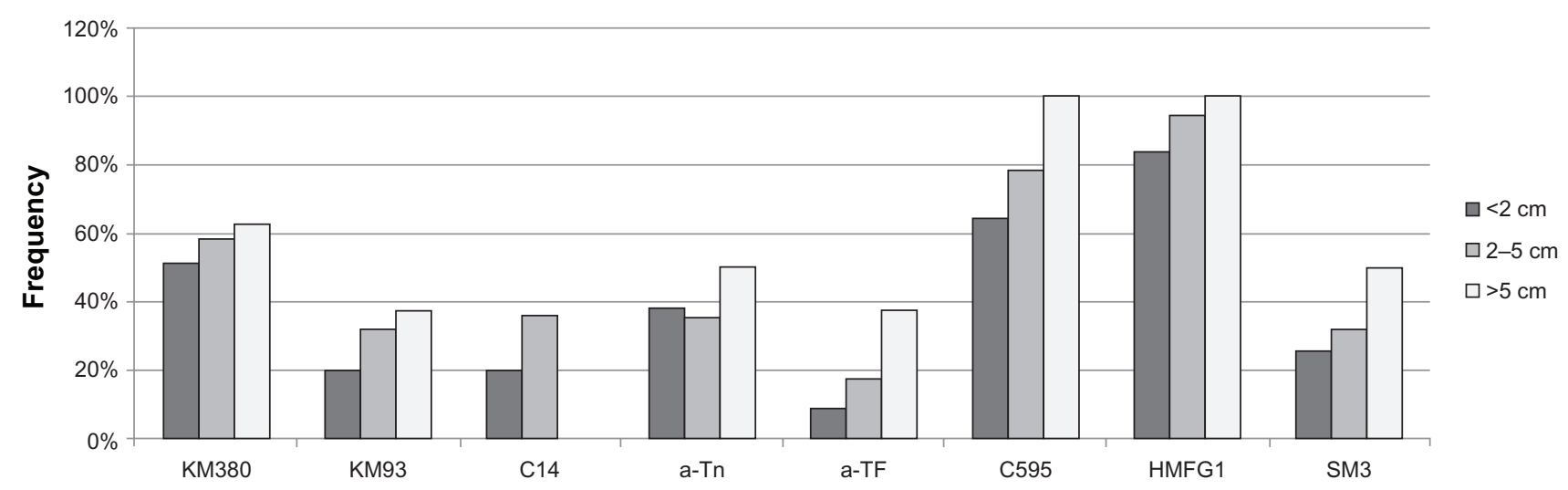

Figure 2 Relative percentage frequencies of staining obtained with the different anticarbohydrate antigens and $\mathrm{MUCl}$ monoclonal antibodies according to tumor size. In all cases, a rising tendency is observed.

A significant mild correlation was found between MUC1 RI detected with HMFG1 MAb and ER ( $r=0.23609$, $P<0.033$ ), whereas with PR status a similar, though not significant, tendency was observed.

\section{Relationship between carbohydrate antigenic expression and prognostic factors}

TF percentage of positive samples increased with disease stage; sialyl Lewis $\mathrm{x}$ as well as Tn expression also augmented with disease stage, although Stage IV showed a decrease, whereas Lewis y and Lewis x did not vary with stage (Table 2).

Stage I patients showed a lower percentage of sialyl Lewis $\mathrm{x}$, Lewis $\mathrm{y}$, and TF reactive area in respect of the other stages $(P<0.011)$. On the contrary, Lewis $\mathrm{x}$ showed a higher percentage of reactive area in Stage I patients and also in more differentiated tumors $(P<0.045)$.

Considering RIs, sialyl Lewis $\mathrm{x}$ and TF RIs increased with disease stage (Figure 4A and B, respectively), showing a positive tendency, whereas Tn RI augmented with the presence of metastatic nodes, histological grade, and mitotic index.

The percentage of glycan-positive responses increased according to tumor size increment, being statistically significant for Tn and Lewis y antigens (Figure 2) $(P<0.0043)$.

\section{Vascular invasion and antigenic expression}

Ejlertsen et $\mathrm{al}^{28}$ defined low-risk patients as those patients who were older than 35 years at diagnosis, with LN-negative disease, tumor size $\leq 2 \mathrm{~cm}$, low or moderate histologic grade, and positive hormonal status. Taking into account this classification, in our study most of the low-risk patients (87\%) did not present vascular invasion (VI), whereas MUC1 apical expression was found in $25 \%$ of them and VI-positive tumors did not show MUC1 apical reaction.

Percentage of positive MUC1 tumor area was higher in VI-negative tumors than in positive ones, being statistically significant when MUC1 was measured with C595 MAb.

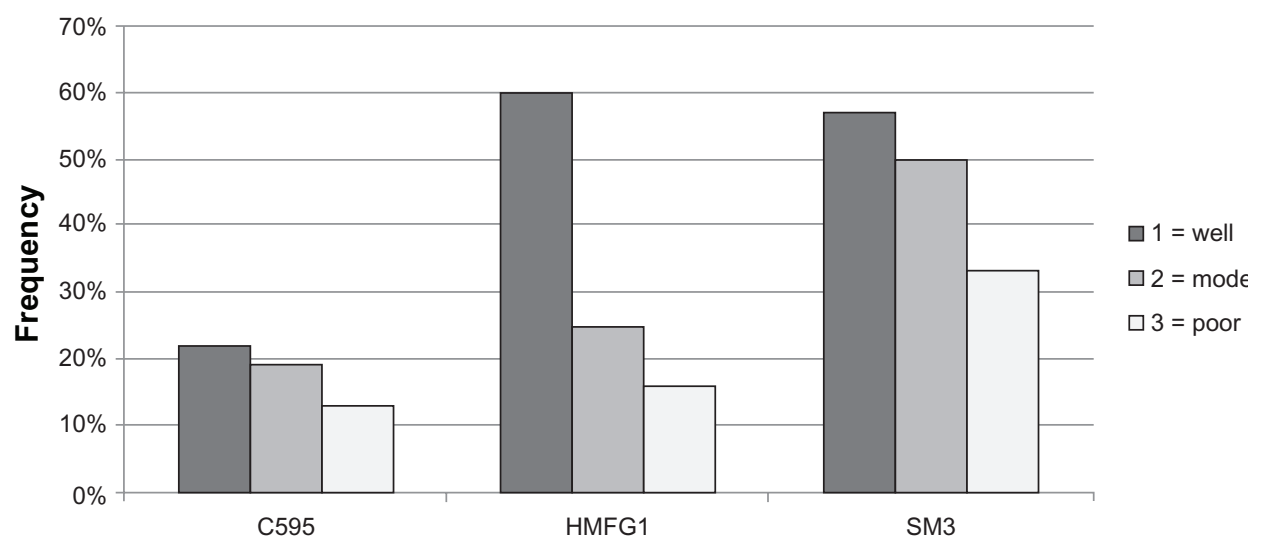

Figure 3 Apical expression frequencies of anti-MUCI monoclonal antibodies according to histological grade. In all cases, apical expression decreases when histological differentiation grades increase. 

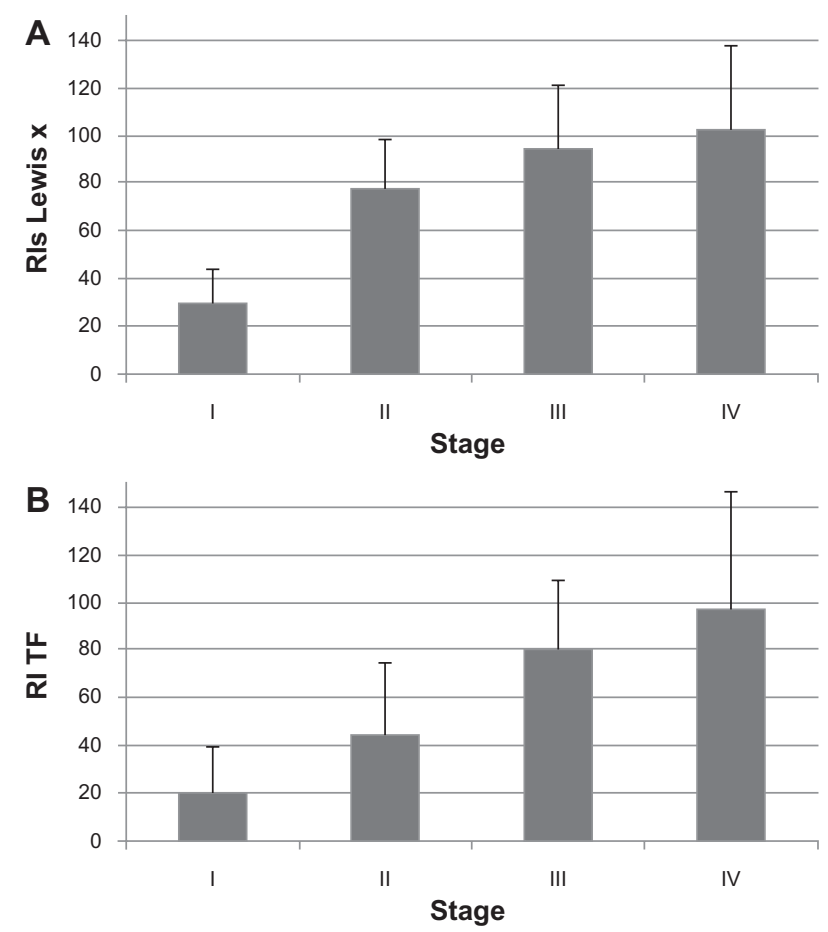

Figure 4 Reactivity index according to disease stage. An increasing reactivity is observed in higher stages: $(\mathbf{A})$ results obtained with anti-sialyl Lewis $x$ monoclonal antibodies (MAbs) and (B) anti-TF MAbs.

Furthermore, poorly differentiated tumors had higher rates of VI than well and moderately differentiated tumors $(P<0.0012)$; in large tumors, VI was detected more frequently. VI was significantly higher in patients aged $<50$ years than in those aged $>50$ years $(P<0.036)$.

Positive tumors for sialyl Lewis $\mathrm{x}$ showed more VI than negative ones: $23 \%$ versus $12 \%(P<0.0073)$. Employing frequency analysis it was possible to demonstrate an association between sialyl Lewis $\mathrm{x}$ expression and the number of metastatic lymph nodes. This analysis showed that patients with sialyl Lewis $x$ reactive primary tumors presented an increment of the frequency on the number of metastatic lymph nodes $(P<0.0249)$. Finally, a positive relationship between VI and Nottingham Prognostic Index was demonstrated $(P<0.033)$.

\section{Relationship among risk factors and antigenic expression}

In relation to the number of children and breastfeeding, the percentage of positive tumor samples in respect of the total showed some variations. When a patient had at least one child, a significant increment on MUC1 reactive area $(P<0.037)$ and MUC1 expression increased significantly from $75 \%$ to $92 \%(P<0.021)$. On the contrary, Lewis antigens diminished their expression in patients who had children, being significant for sialyl Lewis $\mathrm{x}$ (from $46 \%$ to $27 \%, P<0.05$ ) and Lewis y (from $43 \%$ to $22 \%, P<0.025)$. Considering the percentage of MUC1 (detected with HMFG1) reactive area and breastfeeding, a significant increment was found in patients who had breastfed $(P<0.018)$. Although no differences were found between breastfeeding and frequency of positive response, when RI was considered; C595MAb showed a positive tendency, whereas Lewis $\mathrm{x}$ showed a low expression in those patients who had lactated.

In respect of sialyl Lewis $\mathrm{x}$, although its expression decreased according to increase on breastfeeding, its apical expression showed a positive tendency with lactation, whereas Tn and TF antigens showed an increment on apical expression in relation to breastfeeding and number of children $(P<0.018)$.

\section{ER, PR, and Her2-neu subgroups}

In addition, patients were classified according to hormone receptor status (ER and PR) and Her2-neu expression, and analysis in relation to risk and prognostic factors (Table 4) was performed.

Number of positive nodes, tumor size, tumor differentiation, and nuclear grade showed significant differences between ER-positive versus ER-negative subgroups and also between PR-positive versus PR-negative status, whereas age and number of mitotic counts showed significant differences only between ER-positive and -negative subgroups. Furthermore, Her2-neu-positive and Her2-neu-negative patients showed significant differences with number of positive lymph nodes, tumor differentiation, and nuclear grade.

\section{Multivariate analysis}

A multivariate comparative study by PCA was performed, taking into account RI values and risk and prognostic factors (Figure 5). RI corresponding to MUC1 detected with HMFG1 and SM3 MAbs and also to anti-sialyl Lewis $\mathrm{x}$, Lewis $\mathrm{x}$, Lewis y, Tn, and TF explained $59 \%$ of data variability.

When the age was considered, a clear tendency of patients aged $<44$ years to be grouped was observed in PC1(-) and PC2 $( \pm)$ patients, whereas patients aged between 44 years and 70 years showed a spread distribution mainly in PC1(+) and elderly patients ( $>70$ years old) and remained exclusively in $\mathrm{PC} 1(+)$ and $\mathrm{PC} 2(-)$ patients. A similar tendency was observed when menopause status was analyzed. In general, premenopause patients showed less differentiated tumors. In these patients, C595 and HMFG1 anti-MUC1 MAbs showed a very high correlation, HMFG1 being the variable that makes 
Table 4 Logistic regression analysis for patients grouped according to ER, PR, and Her2-neu expression considering risk and prognostic factors

\begin{tabular}{|c|c|c|c|}
\hline \multirow[t]{4}{*}{ Risk and prognostic factors } & \multicolumn{3}{|c|}{ Patient subgoups } \\
\hline & ER & PR & Her2-neu \\
\hline & $P ; O R$ & $P ; O R$ & $P ; O R$ \\
\hline & (ORR CL) & (ORR CL) & (ORR CL) \\
\hline \multirow[t]{2}{*}{ Age (years) } & $0.017 ; 3.53$ & $0.094 ; 2.01$ & $0.921 ; 0.257$ \\
\hline & $(0.916-12.5)^{\mathrm{a}}$ & $(0.070-6.35)$ & $(0.086-1.09)$ \\
\hline \multirow[t]{2}{*}{ Number of children } & $0.224 ; 1.76$ & $0.726 ; 0.527$ & $0.123 ; 1.43$ \\
\hline & $(0.080-1.88)$ & $(0.07 \mid-6.35)$ & $(0.47|-| I .2)$ \\
\hline \multirow[t]{2}{*}{ Breastfeeding } & $0.573 ; 0.415$ & $0.995 ; 0.549$ & $0.848 ; 0.63$ \\
\hline & $(0.095-3.68)$ & $(0.375-2.65)$ & $(0.031-3.16)$ \\
\hline \multirow[t]{2}{*}{ Menopausal age (years) } & $0.099 ; 1.31$ & $0.007 ; 0.126$ & $0.95 \mathrm{I} ; 0.890$ \\
\hline & $(0.008-|.53|)$ & $(0.035-0.416)^{a}$ & $(0.07 I-8.25)$ \\
\hline \multirow[t]{2}{*}{ Menarche age (years) } & $0.685 ; 0.161$ & $0.897 ; 1.13$ & $0.927 ; 0.458$ \\
\hline & $(0.177-13.8)$ & $(0.122-6.34)$ & $(0.066-1.98)$ \\
\hline \multirow[t]{2}{*}{ Disease stage } & $0.214 ; 1.531$ & $0.901 ; 0.849$ & $0.454 ; 2.10$ \\
\hline & $(0.700-1.69)$ & $(0.279-4.24)$ & $(0.028-5.06)$ \\
\hline \multirow[t]{2}{*}{ Number of positive lymph nodes } & $0.002 ; 0.157$ & $0.016 ; 0.219$ & $0.002 ; 15.49$ \\
\hline & $(0.047-0.519)^{\mathrm{a}}$ & $(0.064-0.750)^{\mathrm{a}}$ & $(2.78-86.1)^{\mathrm{a}}$ \\
\hline \multirow[t]{2}{*}{ Tumor size } & $0.004 ; 0.309$ & $0.008 ; 0.349$ & $0.210 ; 1.89$ \\
\hline & $(0.138-0.688)^{\mathrm{a}}$ & $(0.159-0.764)^{\mathrm{a}}$ & $(0.695-5.19)$ \\
\hline \multirow[t]{2}{*}{ Tumor differentiation } & 0,$000 ; 0.274$ & $0.00 \mathrm{I} ; 0.338$ & $0.042 ; 2.95$ \\
\hline & $(0.143-0.525)^{\mathrm{a}}$ & $(0.18 I-0.633)^{\mathrm{a}}$ & $(1.04-8.36)^{\mathrm{a}}$ \\
\hline \multirow[t]{2}{*}{ Nuclear grade } & $0.000 ; 0.237$ & $0.00 \mathrm{I} ; 0.353$ & $0.000 ; 7.83$ \\
\hline & $(0.120-0.467)^{\mathrm{a}}$ & $(0.187-0.665)^{\mathrm{a}}$ & $(1.04-8.36)^{\mathrm{a}}$ \\
\hline \multirow[t]{2}{*}{ Number of mitotic counts } & $0.004 ; 0.093$ & $0.156 ; 0.320$ & $0.434 ; 2.75$ \\
\hline & $(0.019-0.462)^{\mathrm{a}}$ & $(0.066-1.55)$ & $(0.216-34.9)$ \\
\hline \multirow[t]{2}{*}{ Vascular invasion } & $0.890 ; 0.964$ & $0.359 ; 0,790$ & $0.161 ; 1.70$ \\
\hline & $(0.57 I-1.63)$ & $(0.477-1.308)$ & $(0.808-3.59)$ \\
\hline
\end{tabular}

Note: andicates significant regression $P<0.05$.

Abbreviations: $\mathrm{ER}$, estrogen receptor; OR, odds ratio; ORR CL, odds ratio range confidence level for $\pm 95 \%$ range; $\mathrm{PR}$, progesterone receptor.

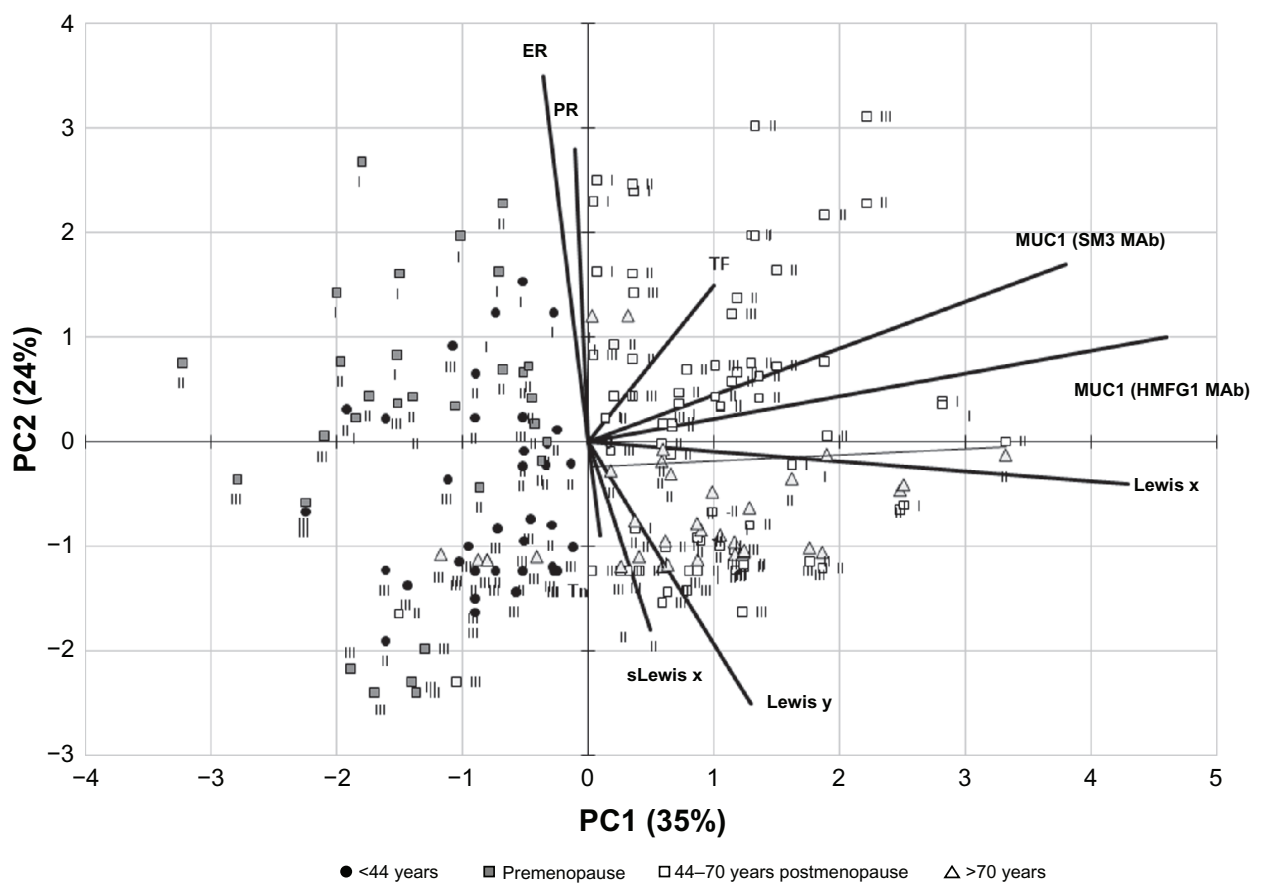

Figure 5 Principal component analysis of reactivity indexes. Length of rays shows variable weights. Points represent each sample data. Patients aged $<44$ years appear grouped on the left of the graphic $\mathrm{PCl}(-)$, whereas middle-age groups remain mainly spread in $\mathrm{PC2}(+)$. Postmenopause and elder patient groups predominate on the right of the graphic $(\mathrm{PCl}(+), \mathrm{PC} 2(+)$ and $\mathrm{PCI}(+), \mathrm{PC2}(-)$, respectively); I, II, and III indicate histological grades. 
the highest contribution; as a consequence, RI obtained with C595 MAb was excluded in the final analysis.

PCA analysis including RI of MUC1 and glycan antigenic expression and also ER and PR separated a group of patients who were characterized by their low age, premenopause status, less differentiated tumors, and negative ER and PR status, PC1(-) and PC2(-).

Employing logistic regression analysis we compared Stage I versus advanced stages using independent variables risk factors. Significant regressions were not found (Table 5).

\section{Benign breast diseases}

Positive results obtained are summarized in Table 2 and depicted in Figure 6A-D. In general, a decrease tendency of positive expression was found from $\mathrm{ADH}$ and $\mathrm{UEH}$ to NPF and FA specimens.

Anti-MUC1 C595 and SM3 MAbs exhibited a very similar pattern of reaction. Apical and linear staining was mainly found.

Lewis $\mathrm{x}$ epitope and sialyl Lewis $\mathrm{x}$ were expressed at apical membranes and cytoplasm. The luminal content was stained. Although the former was found very frequently, the latter was expressed in a low percentage of specimens. Lewis y showed a cytoplasmic mild reaction in several samples, whereas $\mathrm{Tn}$ was reactive in only a few cells in some sections. TF was expressed with either a linear or cytoplasmic pattern, mainly apical and restricted to some areas.

\section{Normal samples}

MUC1 showed a moderate to strong expression. In most samples, an apical pattern mainly restricted to the plasma membrane was found (Figure 6E-F). Lumen content was also stained.

Table 5 Logistic regression analysis for patients grouped according to disease stage using Stage I as a reference and considering risk factors

\begin{tabular}{ll}
\hline Risk and prognostic factors & Stage I vs advanced stages \\
\hline Age (years) & $0.077 ; 0.396$ \\
& $(0.142-1.10)$ \\
Number of children & $0.056 ; 3.7 I$ \\
& $(0.963-14.4)$ \\
Breastfeeding & $0.363 ; 2.70$ \\
& $(0.314-23.5)$ \\
Menopausal age (years) & $0.102 ; 0.261$ \\
& $(0.052-1.31)$ \\
Menarche age (years) & $0.441 ; 1.47$ \\
& $(0.545-3.99)$ \\
\hline
\end{tabular}

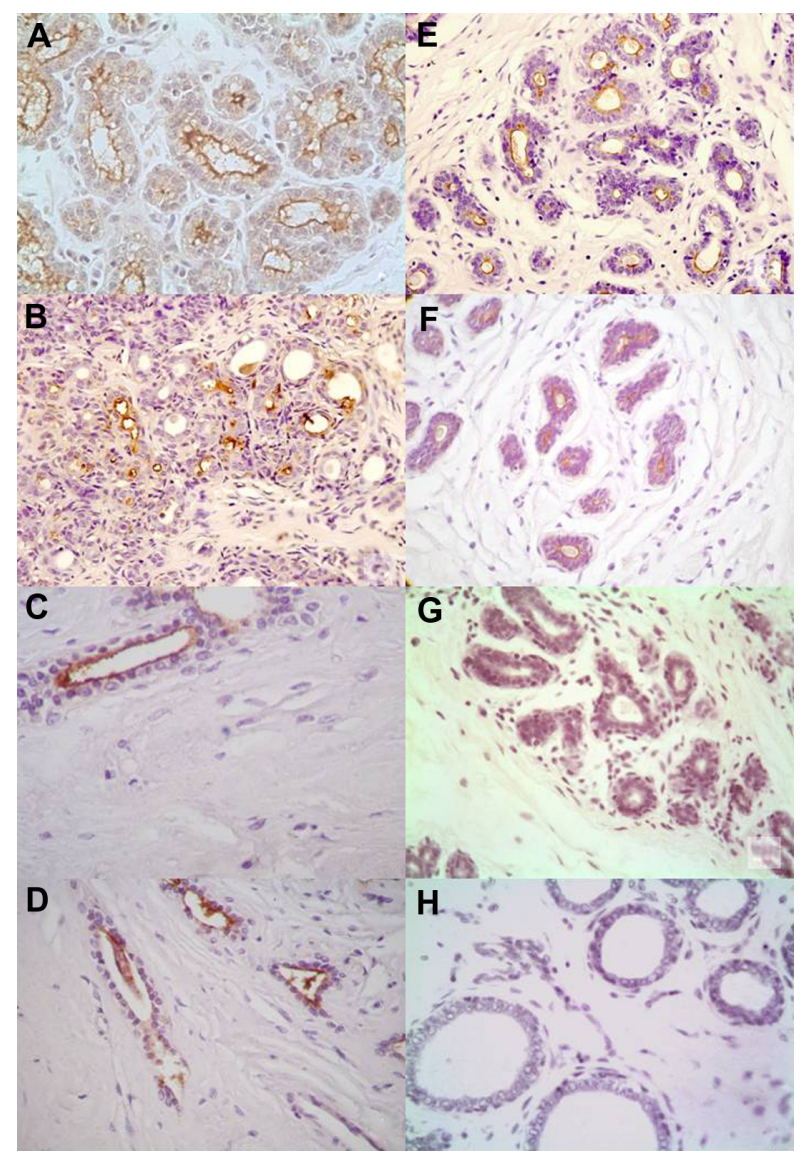

Figure 6 Photomicrographs of immunoperoxidase staining of benign breast diseases (A-D) and normal (E-H) are shown. Staining with anti-MUCl HMFGI monoclonal antibody (MAb), (A) an apical pattern of reaction with a strong intensity is observed in a non proliferative lesion; (E) normal mammary ducts are shown with an apical reactivity. Staining with anti-MUCI C595 MAb, (B) a sample of an apocrine metaplasia is depicted; an apical mainly linear reaction is observed; (F) normal mammary gland sample showing a linear apical reaction. Expression of sialyl Lewis $x(\mathbf{C})$ a strong linear staining in a fibroadenoma sample is shown and $(\mathbf{G})$ a negative normal mammary gland sample is depicted. The TF antigenic reaction in (D) a fibroadenoma specimen is shown with a linear and apical pattern and in a $\mathbf{( H )}$ normal negative sample.

Lewis $\mathrm{x}$ was expressed with a high intensity in the apical part of cell ducts; this pattern was found in all positive samples. Sialyl Lewis x (Figure 6G), Lewis y, and Tn epitopes were detected at the cytoplamic level and with a low intensity, whereas TF was not detected in any sample (Figure 6H).

To compare risk factors between normal group and malignant patients and also between benign versus malignant patients, a logistic regression analysis was performed. Results are summarized in Table 6; significant differences were found only between normal and malignant ages.

In our research, in BBD and normal women, risk factors such as age of menarche, parity, and breastfeeding, were similar to those for breast cancer patients. Significant differences were found between normal and malignant ages (Table 5). 
Table 6 Logistic regression analysis for patients and controls (normal and BBD) considering risk factors.

\begin{tabular}{lll}
\hline $\begin{array}{l}\text { Risk and prognostic } \\
\text { factors }\end{array}$ & $\begin{array}{l}\text { Normal vs malignant } \\
\mathbf{P}(\text { ORR CL) }\end{array}$ & $\begin{array}{l}\text { BBD vs malignant } \\
\mathbf{P}(\text { ORR CL) }\end{array}$ \\
\hline Age (years) & $0.020 ; 128$ & $0.054 ; 3.49$ \\
& $(2.80-61873)^{\mathrm{a}}$ & $(0.947-362)$ \\
Number of children & $0.973 ; 0.956$ & $0.246 ; 20.3$ \\
& $(0.069-13.1)$ & $(0.200-526)$ \\
Breastfeeding & $0.656 ; 0.392$ & $0.389 ; 0.199$ \\
& $(0.006-24.2)$ & $(0.015-5.11)$ \\
Menopausal age (years) & $0.115 ; 10.1$ & $0.276 ; 0.527$ \\
& $(0.567-179)$ & $(0.381-29.1)$ \\
Menarche age (years) & $0.553 ; 2.98$ & $0.742 ; 0.697$ \\
& $(0.081-110)$ & $(0.098-26.2)$ \\
\hline
\end{tabular}

Note: andicates significant regression $P<0.05$.

Abbreviations: $B B D$, benign breast disease; $O R$, odds ratio; $O R R C L$, odds ratio range confidence level for $\pm 95 \%$ range.

This result was expected because normal samples were obtained from women who had had plastic surgery.

\section{Discussion}

Breast cancer studies by area can provide important clues to the underlying factors that influence the biological behavior of this disease. This knowledge would be useful to plan interventions and may serve to define indicators for preventive strategies.

It has been largely described that MUC1 may behave as an oncoprotein. Almost all breast cancer expresses this mucin, although normal breast cells express MUC1 as well. Due to the structural complexity of the MUC1 glycoprotein, multiple epitopes can be detected by monoclonal antibodies. ${ }^{29}$ In this study we found a direct relationship between the percentage of MUC1-positive responses in respect of tumor size, between RI with disease stage, and also between the percentage of positive area and nuclear grade. Coincident with these results we found a significant decrease on MUC1 apical expression frequency according to histological as well as nuclear grade increment. Rakha et $a l^{30}$ pointed out that histological grade can accurately predict tumor behavior. Rahn et a ${ }^{19}$ reported that aberrantly localized MUC1 in the tumor cell cytoplasm or nonapical membrane is associated with a worse prognosis. The presence of apical membrane staining would indicate that MUC1 targeting pathways are intact, and it has been associated with better prognosis possibly related to functional differentiation of the tumor. ${ }^{30}$ Rahn et $a{ }^{19}$ found a significantly lower mean nuclear grade in tumors with high MUC1 expression ( $>50 \%)$. Our results showed a positive relationship between vascular invasion and Nottingham Prognostic Index $(P<0.05)$, and poorly differentiated tumors had higher rates of vascular invasion than well and moderately differentiated tumors $(P<0.005)$. In large tumors, vascular invasion was detected more frequently.

Some authors argue that the glycosylation pattern of MUC1 expressed by normal mammary epithelium is based on elongated Core 2 structures, whereas in breast carcinomas the glycans expressed on MUC1 are often shorter and Core 1 based. ${ }^{31}$ In contrast, Müller and Hanisch ${ }^{32}$ found that no general pattern could be regarded as breast cancer associated and demonstrated a preponderance of Core 2-based structures. These structures have been associated with sialyl Lewis $\mathrm{x}$ determinant, ${ }^{33}$ which acts as a ligand for binding carcinoma cells to endothelium in different tumor localizations, including breast cancer. ${ }^{34,35}$ In this sense, we found that tumors with sialyl Lewis $\mathrm{x}$ expression frequently showed more vascular invasion. This parameter was evaluated by conventional histological assessment, which is considered an adequate method of examination. ${ }^{36}$ Furthermore, we found that patients whose tumors expressed more sialyl Lewis $\mathrm{x}$ had a large number of metastatic lymph nodes $(P<0.01)$ and that patients with sialyl Lewis $\mathrm{x}$ reactive primary tumors presented an increment of the frequency on the number of metastatic lymph nodes $(P>0.05)$. However, we found that sialyl Lewis $\mathrm{x}$ expression was reduced in lymph nodes compared with the primary tumor. This finding coincides with that of Jeschke et al..$^{37}$

In a study that comprised more than 16,000 patients, Ejlertsen et $\mathrm{al}^{28}$ defined low-risk patients as those patients who were older than 35 years at diagnosis, with LN-negative disease, a tumor size $\leq 2 \mathrm{~cm}$, and with a positive hormonal status. Taking into account this classification, in our study most of the low-risk patients (87\%) did not present vascular invasion, whereas MUC1 apical expression was found in $25 \%$ of them. Vascular invasion-positive tumors did not show MUC1 apical reaction at all. This would be related to a defective MUC1 pathway that is associated with lack of functional differentiation and worse prognosis. ${ }^{19}$

It is considered that MUC1 is one of the molecules bearing the epitope sialyl Lewis $\mathrm{x}$ in cancer cells, ${ }^{38}$ as well as other carbohydrate epitopes such as Lewis $\mathrm{x}$ and Lewis $y .{ }^{39}$ In this report, a positive correlation between MUC1 versus Lewis $\mathrm{x}(r=0.8830 ; P<0.05)$ was found and also a tendency between MUC1 and Lewis $\mathrm{x}$ apical expression. These results indicate that MUC1 and Lewis $\mathrm{x}$ would be coexpressed, which would suggest that this mucin may be a Lewis $x$ carrier. In accordance with Brooks and Leathem, ${ }^{40}$ we have detected a higher Lewis $\mathrm{x}$ expression in normal and benign samples than in breast cancer samples. 
In all cases, we observed a strong, mainly apical, staining, although no association with histological grade was found. However, Lewis $\mathrm{x}$ showed a higher percentage of reactive area in more differentiated tumors $(P<0.05)$. Brooks and Leathem ${ }^{40}$ found Lewis $\mathrm{x}$ expression to be associated with invading tumor areas, whereas Elola et $\mathrm{al}^{41}$ confirmed that MCF-7 cancer cells binding to HUVEC partially depended on Lewis x epitopes.

Overexpression of Lewis y antigen has frequently been found in human cancers ${ }^{42}$ and has been observed to be associated with poor prognosis. ${ }^{43}$ Our results showed that the percentage of Lewis y-positive responses increased according to tumor size increment $(P<0.05)$ with different patterns, and many positive samples showed a linear expression. Basu et $\mathrm{al}^{44}$ observed Lewis y at the plasma membrane either as a glycolipid or glycoprotein or linked to surface receptors, such as epidermal growth factor receptors. On the other hand, Klinger et $\mathrm{al}^{45}$ showed that two antiLewis y antibodies inhibited the ErbB-induced stimulation of downstream signalling by intracellular rerouting of internalized EGF receptors.

Tn and TF are early stage-specific differentiation and carcinoma-associated antigens that are related to alterations on mucin type $O$-linked glycosylation and often result in shorter glycan chains. ${ }^{31}$ In accordance to other authors, ${ }^{46}$ our results showed that TF and Tn antigens were expressed in hyperplastic benign diseases and also that, in cancer, their expression showed a positive association with disease stage. Furthermore, the percentage of positive responses augmented in relation to increased tumor size. Employing multivariate analysis, Tsuchiya et $\mathrm{al}^{7}$ found that Tn expression correlated significantly with tumor size, nodal status, and overall survival. Although it has been suggested that Tn and TF antigens may constitute cancer prognostic factors and that they may play significant roles in cancer development, their implications need further investigation.

In this study we analyzed several parameters related to hormonal aspects and antigenic expression. In this sense, we found a significant correlation between MUC1 RI and ER status. This positive correlation was found in the majority of studies previously published, although considering other parameters such as the percentage of tumor cells. ${ }^{16}$ Furthermore, subgroup analysis according to hormone receptor (ER and PR) and antigenic expression showed that ER-positive and PR-positive groups presented higher MUC1 RI than ER-negative and PR-negative groups (statistically different). It is known that ER-positive tumors have better prognosis than negative ones, and almost all tumors expressed MUC1. There is evidence that steroid hormones play a role in MUC1 gene and protein expression. In this sense, McGuckin et $\mathrm{al}^{47}$ found that progesterone caused significant increases in cells and secreted MUC1 in breast cancer cell lines. Moreover, in MCF7 cells, Ren et $\mathrm{al}^{48}$ concluded that estradiol treatment resulted in a gradual increase in MUC1 mRNA expression. Our results showed that with breastfeeding and when a patient had at least one child, MUC1 expression increased. Also, with breastfeeding, antigens involved in tumor dissemination here studied diminished their expression, although their apical pattern increased, suggesting a more differentiated phenotype. It is very interesting that our PCA analysis including RI of MUC1 and glycan antigenic expression and ER and PR separated a group of patients who were characterized by their low age, premenopause status, less differentiated tumors, and more advanced disease stages. In this analysis, which explained $59 \%$ of data variability, menopause status appeared as a relevant factor, because premenopause patients showed less differentiated tumors. When ER and PR were included in this study, premenopause patients with the lowest values were separated. It is considered that exposure of ovarian hormones throughout life affects the risk of developing breast cancer. A metaanalysis of 47 epidemiological studies conducted in 30 countries concluded that the relative risk of breast cancer decreased by $4.3 \%$ (95\% confidence interval 2.9-5.8; $P<0.00001)$ for every 12 months of breastfeeding in addition to a decrease of $7.0 \%(5.0-9.0 ; P<0.0001)$ for each birth. ${ }^{49}$

Tumor cell membrane immunoreactivity for c-erbB2 (Her2-neu) is associated with poorer survival and is more frequently seen in Grade III tumors. ${ }^{50}$ In our analysis, the Her2-neu+ subgroup presented a large number of positive nodes, less differentiated tumors, and higher nuclear grades than in the Her2-neu negative subgroup. Furthermore, it is interesting that Her2-neu+ status showed a significant association with sialyl Lewis $\mathrm{x}$ expression.

The interpretation of breast cancer incidence and mortality patterns is complex in view of the numerous and interactive known and putative risk determinants. In this sense, our results emphasize that the carbohydrate antigenic relationship with risk and prognostic factors contributes to explain breast cancer biological heterogeneity.

The relationship between the carbohydrate antigens selected and risk factors such as breastfeeding and number of children in this malignancy is of special significance, as these factors have been associated with breast protection. The 
panel of antigens studied has been related to tumor progression and dissemination.

\section{Acknowledgments}

Grateful acknowledgement is expressed to Professor J TaylorPapadimitriou and Dr J Burchell (Research Oncology, King's College London, Guy's Hospital, London, UK) for the generous gift of SM3 and HMFG1 MAbs, and to Dr Hanai (Kyowa Hakko Tokyo Res Lab, Tokyo, Japan) for KM380 and KM93 MAbs. Many thanks are expressed to Mr Juan Carlos Molina for technical assistance. Supported by the Comisión de Investigaciones Científicas de la Provincia de Buenos Aires (CIC/PBA), the National University of La Plata, Argentina, and the Kennedy University of Argentina, Buenos Aires, Argentina.

\section{Disclosure}

Professor María Virginia Croce and Dr Marina Isla-Larrain are members of the research team of the Comisión de Investigaciones Científicas de la Provincia de Buenos Aires (CIC/PBA). Professor Sandra O Demichelis is a scientific researcher for the Faculty of Natural Sciences and Museum, the National University of La Plata, and the Department of Biology, Kennedy University of Argentina, Buenos Aires, Argentina.

\section{References}

1. Ferlay J, Shin H-R, Bray F, Forman D, Mathers C, Parkin DM. Estimates of worldwide burden of cancer in 2008: GLOBOCAN 2008. Int $J$ Cancer. 2010;127(12):2893-2917.

2. Ligtenberg MJ, Kruijshaar L, Bujis F, van Meijer M, Litvinov SV, Hilkens J. Cell-associated episalin is a complex containing two proteins derived from a common precursor. J Biol Chem. 1992; 267(9):6171-6177.

3. Gendler S, Taylor-Papadimitriou J, Duhig T, Rothbar J, Burchell J. A highly immunogenic region of a human polymorphic epithelial mucin expressed by carcinomas is made up of tandem repeats. $J$ Biol Chem.1988;263(26):12820-12823.

4. Merlo G, Siddiqui J, Cropp C, et al. DF3 tumor-associated antigen gene is located in a region on chromosome 1q frequently altered in primary human breast cancer. Cancer Res. 1989(24);40:6966-6971.

5. McGuckin MA, Quinn RJ, Ward BG. Progesterone stimulates production and secretion of MUC1 epithelial mucin in steroid-responsive breast cancer cell lines. Int J Oncol. 1998;12:939-945.

6. Gendler S. MUC1, the renaissance molecule. J Mamm Gland Biol and Neoplasia. 2001;6(3):339-353.

7. Tsuchiya A, Kanno M, Kawaguchi T, et al. Prognostic relevance of Tn expression in breast cancer. Breast Cancer. 1999;6(3):175-180.

8. Hakomori S. Tumor malignancy defined by aberrant glycosylation and sphingo(glyco)lipid metabolism. Cancer Res. 1996;56(23):5309-5318.

9. Bernstein L. Epidemiology of endocrine-related risk factors for breast cancer. J Mamm Gland Biol and Neoplasia. 2002;7:3-15.

10. Russo J, Moral R, Balogh GA, Mailo D, Russo IH. The protective role of pregnancy in breast cancer. Breast Cancer Res. 2005;7: 131-142.
11. Burchell J, Gendler S, Taylor-Papadimitriou J, et al. Development and characterization of breast cancer reactive monoclonal antibodies directed to the core protein of the human milk mucin. Cancer Res. 1987;47(20):5476-5482.

12. Devine P, McGuckin M, Quinn R, Ward B. Serum CASA and CA 15-13 in ovarian cancer: all MUC1 assays are not the same. Tumor Biol. 1994;15(6):337-344.

13. Croce MV, Isla Larrain MT, Capafons A, Price MR, Segal-Eiras A. Humoral immune response induced by protein core of MUC1 mucin in pregnant and healthy women. Breast Cancer Res Treat. 2001;2044:1-11.

14. Agrawal B, Krantz MJ, Parker, Longenecker M, Kenemans P, Bast RC, et al. Mucins and mucin-like antigens as circulating tumor markers. In: Hilgers J, Zotter S, eds. Cancer Review, Vol 11, 12. Copenhagen, Demark: Munksgaard; 1989: 19-44.

15. Croce MV, Isla Larrain MT, Price MR, Segal-Eiras A. Detection of circulating mammary mucin (MUC1) and MUC1 immune complexes (MUC1-CIC) in healthy women. Int J Biol Markers. 2001;16(2): $112-120$.

16. Rasmussen BB, Pedersen BV, Thorpe SM, Hilkens J, Hilgers J, Rose C. Prognostic value of surface antigens in primary human breast carcinomas, detected by monoclonal antibodies. Cancer Res. 1985;45(3):1424-1427.

17. Ellis IO, Hinton CP, MacNay J, et al. Immunocytochemical staining of breast carcinoma with the monoclonal antibody NCRC 11: a new prognostic indicator. Br Med J. 1985;290(6472):881-883.

18. Baildman AD, Howell A, Barnes DM, Turnbull L, Sellwood RA. The expression of milk fat globule antigens within human mammary tumors: relationship to steroid hormone receptors and response to endocrine treatment. Eur J Cancer. 1989;25(3):459-467.

19. Rahn J, Dabbagh L, Pasdar M, Hugh J. The importance of MUC1 cellular localization in patients with breast carcinoma. Cancer. 2001;91(11):1973-1982.

20. Elston CW, Ellis IO. Pathological prognostic factors in breast cancer. I. The value of histological grade in breast cancer: experience from a large study with long-term follow-up. Histopathol. 1991;19(5): 403-410.

21. Taylor-Papadimitriou J, Peterson JA, Arklie J, Burchell J, Ceriani RL, Bodmer WF. Monoclonal antibodies to epithelium specific components of the milk fat globule membrane: production and reactions with cells in culture. Int J Cancer. 1981;28(1):17-21.

22. Price MR, Pugh JA, Hudecz F, Griffiths W, Jacobs E, Symonds IM, et al. C595: a monoclonal antibody against the protein core of human urinary epithelial mucin commonly expressed in breast carcinomas. Br J Cancer. 1990;61(5):681-686.

23. Hanai N, Shitara K, Yoshida H. Generation of monoclonal antibodies against human lung squamous cell carcinoma and adenocarcinoma using mice rendered tolerant to normal human lung. Cancer Res. 1986;46(9):4438-4443.

24. Brown A, Feizi T, Gooi HC, Embleton MJ, Baldwin R. A monoclonal antibody against human colonic adenoma recognizes difucosylated type-2-blood-group chains. Biosci Rep. 1983;3(2):163-170.

25. Croce MV, Isla Larrain MT, Remes-Lenicov F, et al. MUC1 cytoplasmic tail detection using CT33 polyclonal and CT2 monoclonal antibodies in breast and colorectal tissue. Histol and Histopathol. 2006;21(8):849-855.

26. Demichelis S, Alberdi C, Servi W, Isla Larrain MT, Segal-Eiras A, Croce MV. Comparative immunohistochemical study of MUC1 and carbohydrate antigens in breast benign disease and normal mammary gland. Appl Immunohistochem Mol Morphol. 2010;18(1): $41-50$.

27. Croce MV, Isla-Larrain M, Demichelis, S, Gori J, Price MR, Segal-Eiras A. Tissue and serum MUC1 detection in breast cancer patients. Breast Cancer Res Treat. 2003;81(3):195-207.

28. Ejlertsen B, Jensen M, Rank F, et al. Population-based study of peritumoral lymphovascular invasion and outcome among patients with operable breast cancer. J Nat Cancer Inst. 2009;101(10): 729-735. 
29. Burchell J, Durbin H, Taylor-Papadimitriou J. Complexity of expression of antigenic determinants, recognized by monoclonal antibodies HMFG-1 and HMFG-2, in normal and malignant human mammary epithelial cells. J Immunol. 1983;131(1):508-513.

30. Rakha E, Reis-Filho J, Baehner F, et al. Breast cancer prognostic classification in the molecular era: the role of histological grade. Breast Cancer Res. 2010;12(4):207.

31. Burchell J, Mungul, A, Taylor-Papadimitriou J. O-linked glycosylation in the mammary gland: changes that occur during malignancy. J Mamm Gland Biol Neoplasia. 2001;6(3):355-364.

32. Müller S, Hanisch F-G. Recombinant MUC1 probe authentically reflects cell-specific O-glycosylation profiles of endogenous breast cancer mucin. J Bi. Chem. 2002;277(29):26103-26112.

33. Maemura K, Fukuda M. Poly-N-acetyllactosaminyl 0-glycans attached to leukosialin. J Biol Chem. 1992;267(34):24379-24386.

34. Takada A, Ohmori K, Yoneda T, et al. Contribution of carbohydrate antigens sialyl lewis A and sialyl Lewis $\mathrm{x}$ to adhesion of human cancer cells to vascular endoithelium. Cancer Res. 1993;53(2):354-361.

35. Renkonen J, Paavonen T, Renkonen R. Endothelial and epithelial expression of sialyl Lewis $\mathrm{x}$ and sialyl Lewis a in lesions of breast carcinoma. Int J Cancer. 1997;74(3):296-300.

36. Ito M, Moriy T, Ishida T, et al. Significance of pathological evaluation for lymphatic vessel invasion in invasive breast cancer. Breast Cancer. 2007;14(4):381-387.

37. Jeschke U, Mylonas I, Shabani M, et al. Expression of sialyl Lewis X, sialyl Lewis a, E-cadherin and cathepsin-D in human breast cancer: immunohistochemical analysis in mammary carcinoma in situ, invasive carcinomas and their lymph node metastasis. Anticancer Res. 2005;25(3A):1615-1622.

38. Hanski C, Drechsler K, Hanisch F-G, et al. Altered glycosylation of MUC1 protein core contributes to the colon carcinoma associated increase of mucin-bound sialyl Lewis x expression. Cancer Res. 1993;53(17):4082-4088

39. Isla Larrain M, Demichelis S, Crespo MV, et al. Breast cancer humoral immune response: involvement of Lewis y through the detection of circulating immune complexes and association with Mucin 1 (MUC1) J Exp Clin Cancer Res. 2009;28(1):121.
40. Brooks SA, Leathem AJ. Expression of the CD15 antigen (Lewis $\mathrm{x}$ ) in breast cancer. Histochem J. 1995;27(9):689-693.

41. Elola MT, Capurro MI, Barrio MM, et al. Lewis x antigen mediates adhesion of human breast carcinoma cells to activated endothelium: possible involvement of the endothelial scavenger receptor C-type lectin. Breast Cancer Res Treat. 2007;101(2):161-174.

42. Hellstrom I, Garrigues HJ, Garrigues U, Hellstrom KE. Highly tumorreactive, internalizing, mouse monoclonal antibodies to Le(y)-related cell surface antigens. Cancer Res. 1990;50(7):2183-21905.

43. Yan L, Lin B, Gao L, et al. Lewis (y) antigen overexpression increases the expression of MMP-2 and MMP-9 and invasion of human ovarian cancer cells. Int J Mol Sci. 2010;11(11):4441-4451.

44. Basu A, Murthy U, Rodeck U, Herlyn M, Mattes L, Das M. Presence of tumor-associated antigens in epidermal growth factor receptors from different human carcinomas. Cancer Res. 1987;47(10):2531-2536.

45. Klinger M, Farhan H, Just H, Drobny H, Himmler G, Loibner G, et al. Antibodies directed against Lewis- $Y$ antigen inhibit signaling of lewis- $Y$ modified ErbB receptors. Cancer Res. 2004;64(3):1087-1093.

46. Deutscher SL, Dickerson M, Gui G, et al. Carbohydrate antigens in nipple aspirate fluid predict the presence of atypia and cancer in women requiring diagnostic breast biopsy. BMC Cancer. 2010;10:519.

47. McGuckin MA, Walsh MD, Hohn BG, Ward BG, Wright RG. Prognostic significance of MUC1 epithelial mucin expression in breast cancer. Hum Pathol. 1995;26(4):432-439.

48. Ren L, Marquardt MA, Lech JJ. Estrogenic effects of nonylphenol on $\mathrm{pS} 2$, ER and MUC1 gene expression in human breast cancer cellsMCF-7. Chem Biol Interact. 1997;104(1):55-64.

49. Collaborative Group on Hormonal Factors in Breast Cancer. Breast cancer and breastfeeding: collaborative reanalysis of individual data from 47 epidemiological studies in 30 countries, including 50,302 women with breast cancer and 96,973 women without the disease. Lancet. 2002;360(9328):187-195.

50. Lovekin C, Ellis IA, Locker AP, et al. CerbB2 oncoprotein expression in primary and advanced breast. Br J Cancer. 1991;63:439-441.
Breast Cancer: Targets and Therapy

\section{Publish your work in this journal}

Breast Cancer: Targets and Therapy is an international, peerreviewed open access journal focusing on breast cancer research, identification of therapeutic targets and the optimal use of preventative and integrated treatment interventions to achieve improved outcomes, enhanced survival and quality of life for the cancer patient.

\section{Dovepress}

View the full aims and scopes of this journal here. The manuscript management system is completely online and includes a very quick and fair peer-review system, which is all easy to use. Visit http:// www.dovepress.com/testimonials.php to read real quotes from published authors. 